\title{
Determination of Antioxidant Content and Activity in Eight Jordanian Fresh Green Leafy Vegetables
}

\author{
Hiba Al-Sayyed*, Refa't Al-Kurd, Marwan Mwalla, and Salma Abdel Qader \\ Department of Clinical Nutrition and Dietetics, University of Petra, Jordan
}

Submission: January 12, 2019, Published: January 25, 2019

*Corresponding author: Hiba F Al-Sayyed, Department of Nutrition, Faculty of Pharmacy and Medical Sciences, University of Petra, P.0.BOX. 961343 - 11196, Amman, Jordan

\begin{abstract}
The consumption of vegetables that contain natural antioxidants is thought to be an efficient way for reducing the risk for oxidative stress diseases. Determination of antioxidant content and capacity allows the screening of vegetables that are probably involved in the prevention and/ or treatment of oxidative stress diseases. This study aimed at comparing eight fresh Jordanian green leafy vegetables (namely: grape leaves, lettuce, mint, parsley, Jew's mallow, watercress, celery, and garden rocket) for their antioxidant content (using two methods namely: Folin-Ciocaltaeu method and total flavonoid method) and capacity (using two methods namely: 2,2-diphenyl-picrylhydrazyl (DPPH) and cupric antioxidant reducing capacity (CUPRAC) assays). Three solvents were used for the vegetable extraction (ethanol, methanol, and water). Different solvents as well as different vegetables showed significantly $(\mathrm{P}<0.05)$ different antioxidant contents and capacities. Significant correlations $\left(\mathrm{P}<0.001^{* *} ; \mathrm{r}>0.90\right)$ were found between antioxidant content and capacity of the studied vegetables.
\end{abstract}

Keywords: Jordan; Antioxidants; CUPRAC; DPPH; Folin-Cioculteau; Green leafy vegetables; Total flavonoids

\section{Introduction}

Free-radicals are highly reactive molecules that lost one or more of the electrons of their outermost orbit; enabling them for hitting the electronically stable outermost orbit of a stable atom or an atom of a molecule for the purpose of steeling one of the electrons to be stable. Free radicals are formed continuously within the human body during cell respiration. Simultaneously, the body has many mechanisms to get rid of these highly reactive molecules. When the formation of free radicals exceeds the body capacity to get rid of them, oxidative damage occurs. Oxidative damage is involved in many chronic diseases such as cardiovascular diseases, type I diabetes, cataract, arthritis and certain types of cancer. Antioxidants are substances that, when present at low concentrations -compared to oxidized substratesdelay or prevent that substrate oxidation significantly [1]. The consumption of vegetables that contain natural antioxidants is thought to be an efficient way for reducing the risk for oxidative stress diseases [2]. Determination of antioxidant content and capacity, therefore, allows the screening of vegetables that are probably involved in the prevention and/ or treatment of oxidative stress diseases.

The purpose of this study is to screen eight Jordanian fresh green leafy vegetables that are consumed regularly (namely: grape leaves, lettuce, mint, parsley, Jew's mallow, watercress, celery, and garden rocket) for their antioxidant content and capacity. Additionally, this study aimed to find a correlation between the antioxidant content and capacity of the selected vegetables. This study is probably the first study that evaluated the antioxidant content and capacity of locally grown fresh Jordanian vegetables extracted by three solvents (i,e. ethanol, methanol, and water) in order to add a value to the scientific antioxidant database. In Jordan, there were many attempts to determine the antioxidant content and capacity of Jordanian plants [3-5]. There is -as well- a great interest in plants in Jordan in terms of classification [6], studying of nutritional value and methods of use [7], and functional properties $[8,9]$.

\section{Materials and Methods}

Fresh vegetables were purchased from local market and prepared in the same day. Vegetables were prepared by washing with tap water and gentle drying by towel paper. Samples were then chopped finely by knife or food chopper (Ariete ${ }^{\circledR}$, China). Representative samples (1-3g) were extracted conventionally by $10 \mathrm{ml}$ of one of three extraction solvents (methanol, ethanol, and water) at $50{ }^{\circ} \mathrm{C}, 50{ }^{\circ} \mathrm{C}$, and $90{ }^{\circ} \mathrm{C}$ respectively for 2 hours with intermittent shaking. The extracts were centrifuged at 3000rpm for 10-15 minutes (HuMax®, Germany) and filtered (Wattman filter paper No.4), purged with liquid nitrogen [2], and stored at $-20{ }^{\circ} \mathrm{C}$ (for not more than two months) until analyzed. Deionized water was used for the preparation of all standard solutions and to complete the reactions [2]. 


\section{Determination of antioxidant content}

Chemicals were purchased from GCC $\AA$ (UK), Fischer ${ }^{\circledR}$ (China), Labscan ${ }^{\circledR}$ (Thaihland), LabChem ${ }^{\circledR}$ (USA) and Sigma ${ }^{\circledR}$ (China). Standard curves were prepared to have $r^{2}$ value of 0.96-0.99. Samples were analyzed in duplicate with an accuracy of not less than $95 \%$ [10] and coefficient of variation not more than $15 \%$. Absorbance values were measured using UV-visible spectrophotometer (SCO Tech, Model SPUV®) at the specified wavelength values against standard concentrations of certain antioxidants and blank solutions.

Folin-ciocultaeu method: Folin-Ciocultaeu method was used for the determination of antioxidant content according to Agbor et al. [11]. Sample concentration for antioxidants was measured against freshly prepared catechin standard at $750 \mathrm{~nm}$ wavelength.

Total flavonoid method: Total flavonoids were analyzed by the method of Pękal and Pyrzynka [12]. The absorbance was measured at $400 \mathrm{~nm}$ wavelength against different concentrations of rutin standard solutions.

\section{Determination of antioxidant capacity}

CUPRAC Assay: Sample antioxidant capacity was measured by CUPRAC assay according to Apak et.al. [2]. Absorbance was Results and Discussion

Table 1: The antioxidant content of the ethanolic, methanolic, and water vegetable extracts determined by Folin-Ciocultaeu method (M catechin/100g) and total flavonoid method (M rutin/100g) $)^{1,2}$

\begin{tabular}{|c|c|c|c|c|c|c|c|}
\hline \multirow{3}{*}{ Vegetable } & \multicolumn{3}{|c|}{ Folin-Ciocultaeu Method } & \multicolumn{4}{|c|}{ Total Flavonoid Method } \\
\hline & \multicolumn{3}{|c|}{ Extract } & \multicolumn{4}{|c|}{ Extract } \\
\hline & Ethanol & Methanol & Water & Ethanol & Methanol & Water & P-value \\
\hline Celery & $0.9533 \pm 0.0265$ & $0.6274 \pm 0.0773$ & $0.9533 \pm 0.0265$ & $0.4809 \pm 0.0524$ & $0.3587 \pm 0.0088$ & $0.1738 \pm 0.0092$ & $\begin{array}{c}<0.01^{* *} \\
\text { (Folin- } \\
\text { Ciocultaeu } \\
\text { method) }\end{array}$ \\
\hline $\begin{array}{l}\text { Garden } \\
\text { rocket }\end{array}$ & $3.5563 \pm 0.4517$ & $1.3714 \pm 0.0519$ & $3.4257 \pm 0.3939$ & $0.8478 \pm 0.0075$ & $0.3366 \pm 0.0038$ & $0.1669 \pm 0.0021$ & $\begin{array}{c}<0.01^{* *} \\
\text { (Total } \\
\text { Flavanoids } \\
\text { Method) }\end{array}$ \\
\hline $\begin{array}{l}\text { Grape } \\
\text { leaves }\end{array}$ & $74.4792 \pm 1.8382$ & $22.4282 \pm 0.3143$ & $29.7666 \pm 1.3531$ & $1.1103 \pm 0.0699$ & $0.7320 \pm 0.0133$ & $0.7168 \pm 0.0516$ & \\
\hline Lettuce & $1.1869 \pm 0.0227$ & $0.9023 \pm 0.0218$ & $1.4208 \pm 0.0288$ & $0.0287 \pm 0.0043$ & $0.0267 \pm 0.0007$ & $0.02110 \pm 0.0021$ & \\
\hline Mint & $77.6903 \pm 9.6764$ & $24.1357 \pm 0.3320$ & $45.0805 \pm 1.3579$ & $2.9404 \pm 0.0476$ & $3.1849 \pm 0.01210$ & $1.1894 \pm 0.0291$ & \\
\hline Parsley & $10.9093 \pm 1.0836$ & $8.9465 \pm 0.7487$ & $10.5018 \pm 0.7495$ & $5.3971 \pm 0.4555$ & $4.8651 \pm 0.3241$ & $1.1349 \pm 0.0297$ & \\
\hline $\begin{array}{l}\text { Jew's } \\
\text { Mallow }\end{array}$ & $12.1150 \pm 0.1114$ & $14.8060 \pm 0.3275$ & $23.7710 \pm 0.9788$ & $0.1090 \pm 0.0107$ & $1.0704 \pm 0.0449$ & $0.1512 \pm 0.0050$ & \\
\hline Watercress & $2.8772 \pm 0.2092$ & $1.8392 \pm 0.1401$ & $2.3765 \pm 0.0235$ & $0.4103 \pm 0.0159$ & $0.1824 \pm 0.0055$ & $0.0042 \pm 0.0002$ & \\
\hline
\end{tabular}

${ }^{1}$ Values of the tables are average of duplicates \pm SEM with c.v. of not more than $15 \%$.

${ }^{2} \mathrm{P}$ values are used to express significant differences between different vegetable extracts.

Table 1 shows the antioxidant content determined by Folin-Ciocultaeu and total flavonoid methods for the ethanolic, methanolic, and water vegetable extracts. Regardless of the extraction solvent; the antioxidant content (expressed as $\mathrm{M}$ catechin/100 g sample) was the highest $(\mathrm{P}<0.05)$ for mint>grape leaves $>$ parsley $>$ Jew's mallow $>$ garden rocket. Nonetheless, there measured using a spectrophotometer at 450nm [2] against different concentrations of trolox standard solutions.

DPPH assay: The DPPH assay procedure was performed according to Molyneux [13]. The absorbance was measured at $517 \mathrm{~nm}$ wavelength against ascorbic acid as a standard. The scavenging percentage was calculated according to the following equation:

Scavenging effect $(\%)=\left(\left(A_{0}-A_{1}\right) / A_{0}\right) * 100 \%$

Where: $A_{0}$ : absorbance of the control; $A_{1}$ : absorbance of the sample

\section{Statistical Analysis}

The statistical analysis of data was performed using the software package for social sciences (SPSS, version 23). To detect the differences between the 8 different vegetables as well as the extraction solvent, data were analyzed by factorial mixed (effect of type of plant and extracts types) analysis of variance (ANOVA) [14]. Significant differences were considered at $\mathrm{P}<0.05$. Data are expressed in the tables as mean \pm standard deviation. Pearson's correlation coefficient was calculated and considered significant at $\mathrm{P}<0.05$. 
from mint, grape leaves, parsley, garden rocket, watercress, and celery than methanol $(\mathrm{P}<0.05)$. From Jew's mallow only, water followed by $(\mathrm{P}<0.05)$ methanol had extracted higher $(\mathrm{P}<0.05)$ amounts of antioxidants than ethanol did. From lettuce only, water followed by $(\mathrm{P}<0.05)$ ethanol extracted higher amounts $(\mathrm{p}<0.05)$ of antioxidants than methanol did.

Regardless of the extraction solvent, parsley> mint>grape leaves $>$ garden rocket $>$ lettuce had the highest $(\mathrm{P}<0.05)$ antioxidant contents (Mrutin/100g). In terms of the extraction solvent, ethanol followed by methanol $(\mathrm{P}<0.05)$ had extracted more antioxidants $(\mathrm{P}<0.05)$ than water from parsley, grape leaves, garden rocket, celery, watercress, and lettuce. Methanol followed by ethanol $(\mathrm{P}<0.05)$ had extracted more antioxidants than water from mint. On the other hand, methanol followed by water $(\mathrm{P}<0.05)$ had extracted more antioxidants than ethanol from Jew's mallow.
Table 2 shows the antioxidant capacity determined by CUPRAC assay for the ethanolic, methanolic, and water vegetables extracts. Regardless of the extraction solvent, watercress $>$ mint $>$ grape leaves $>$ lettuce $>$ parsley $>$ Jew's mallow $>$ celery had the highest antioxidant capacity values. In terms of the extraction solvent, ethanol followed by methanol $(\mathrm{P}<0.05)$ had extracted antioxidants more powerfully $(\mathrm{P}<0.05)$ than water from parsley, garden rocket, and celery. Methanol followed by $(\mathrm{P}<0.05)$ water had extracted antioxidants more $(\mathrm{P}<0.05)$ powerfully than ethanol from Jew's mallow and watercress. On the other hand, water followed by methanol $(\mathrm{P}<0.05)$ had extracted antioxidants more powerfully $(\mathrm{P}<0.05)$ than ethanol from mint and grape leaves. Water followed by methanol $(\mathrm{P}<0.05)$ had extracted antioxidants more $(\mathrm{P}<0.05)$ powerfully than ethanol from mint and grape leaves. From lettuce, water followed by ethanol $(\mathrm{P}<0.05)$ had extracted antioxidants more $(\mathrm{P}<0.05)$ powerfully than methanol did.

Table 2: The antioxidant capacity (M trolox/100g) of the ethanolic, methanolic, and water vegetable extracts determined by CUPRAC assay ${ }^{1,2}$

\begin{tabular}{|c|c|c|c|}
\hline Vegetable & \multicolumn{2}{|c|}{ Antioxidant Capacity (M trolox/100g) as Determined by Total CUPRAC Assay } \\
\hline & \multicolumn{2}{|c|}{ Extract } \\
\hline & Ethanol & $1.1155 \pm 0.0391$ & $1.0298 \pm 0.0165$ \\
\hline Celery & $1.7725 \pm 0.2361$ & $3.7643 \pm 0.017$ & $2.2329 \pm 0.1187$ \\
\hline Garden rocket & $3.8115 \pm 0.0786$ & $18.4574 \pm 0.1032$ & $53.6694 \pm 1.3151$ \\
\hline Grape leaves & $17.9871 \pm 0.3409$ & $0.54944 \pm 0.0253$ & $2.4399 \pm 0.2667$ \\
\hline Lettuce & $0.6584 \pm 0.0000$ & $23.8290 \pm 1.5477$ & $23.9893 \pm 2.8210$ \\
\hline Mint & $20.5841 \pm 0.4903$ & $6.4861 \pm 0.1226$ & $4.1143 \pm 0.2099$ \\
\hline Parsley & $11.5835 \pm 0.0162$ & $15.8392 \pm 0.3314$ & $15.0906 \pm 0.7481$ \\
\hline Jew's mallow & $9.8804 \pm 0.2549$ & $3.4036 \pm 0.24078$ & $2.3272 \pm 0.0648$ \\
\hline Watercress & $2.2432 \pm 0.1172$ & & \\
\hline
\end{tabular}

${ }^{1}$ Values of the tables are average of duplicates \pm SEM with c.v. of not more than $15 \%$.

${ }^{2} \mathrm{P}$ values are used to express significant differences between different vegetable extracts.

Table 3: The antioxidant capacity of the ethanolic, methanolic, and water vegetable extracts determined by DPPH assay (expressed as \% of DPPH radical scavenging and $\mathrm{mg}$ vitamin $\mathrm{C} / \mathrm{ml}$ extract $)^{1,2}$.

\begin{tabular}{|c|c|c|c|c|c|c|c|}
\hline \multirow{3}{*}{ Vegetable } & \multicolumn{3}{|c|}{ \% DPPH scavenging } & \multicolumn{4}{|c|}{$\mathrm{mg}$ vitamin $\mathrm{C} / \mathrm{ml}$} \\
\hline & \multicolumn{3}{|c|}{ Extract } & \multicolumn{4}{|c|}{ Extract } \\
\hline & Ethanol & Methanol & Water & Ethanol & Methanol & Water & P-value \\
\hline Celery & $4.823 \pm 0.455$ & $15.264 \pm 2.060$ & $17.546 \pm 0.056$ & $59.915 \pm 5.649$ & $194.946 \pm 26.300$ & $161.8631 \pm 0.521$ & $\begin{array}{c}0.000^{* *} \\
\text { (\% DPPH } \\
\text { scavenging) }\end{array}$ \\
\hline $\begin{array}{c}\text { Garden } \\
\text { rocket }\end{array}$ & $11.362 \pm 1.566$ & $14.441 \pm 0.211$ & $18.745 \pm 0.754$ & $92.598 \pm 12.764$ & $93.712 \pm 1.425$ & $111.856 \pm 4.275$ & $\begin{array}{c}0.000^{* *}(\mathrm{mg} \\
\text { vitamin } \mathrm{C} / \mathrm{ml} \\
\text { extract) }\end{array}$ \\
\hline Grape leaves & $35.128 \pm 1.583$ & $57.981 \pm 0.506$ & $88.426 \pm 0.982$ & $213.543 \pm 9.624$ & $439.470 \pm 3.595$ & $506.874 \pm 5.567$ & \\
\hline Lettuce & $9.220 \pm 0.134$ & $28.465 \pm 0.390$ & $21.805 \pm 2.528$ & $56.052 \pm 0.817$ & $184.715 \pm 2.529$ & $129.2051 \pm 14.329$ & \\
\hline Mint & $25.289 \pm 0.204$ & $50.184 \pm 0.676$ & $88.209 \pm 0.2111$ & $206.104 \pm 1.666$ & $384.090 \pm 4.800$ & $428.4068 \pm 1.025$ & \\
\hline Parsley & $7.298 \pm 0.818$ & $6.066 \pm 1.196$ & $14.129 \pm 1.267$ & $59.476 \pm 6.662$ & $70.754 \pm 8.493$ & $68.6224 \pm 6.151$ & \\
\hline Jew's mallow & $12.808 \pm 0.257$ & $15.399 \pm 3.355$ & $36.319 \pm 0.689$ & $104.388 \pm 2.091$ & $137.040 \pm 23.824$ & $176.393 \pm 3.344$ & \\
\hline Watercress & $29.020 \pm 0.561$ & $23.463 \pm 0.424$ & $39.667 \pm 4.687$ & $236.508 \pm 4.570$ & $194.317 \pm 3.012$ & $192.660 \pm 22.761$ & \\
\hline
\end{tabular}

${ }^{1}$ Values of the tables are average of duplicates \pm SEM with c.v. of not more than $15 \%$.

${ }^{2} \mathrm{P}$ values are used to express significant differences between different vegetable extracts.

Table 3 shows the antioxidant capacity determined by DPPH assay and expressed as \% DPPH scavenging and as mg vitamin $\mathrm{C} / \mathrm{ml}$ extract. Regardless of the extraction solvent, the highest

antioxidants capacities were for grape leaves $>$ mint $>$ watercress $>$ Jew's mallow> garden rocket>parsley. In terms of the extraction solvent, water followed by methanol $(\mathrm{P}<0.05)$ had extracted 
antioxidants more powerfully $(\mathrm{P}<0.05)$ than ethanol from grape leaves, mint, Jew's mallow, garden rocket, and celery. Methanol followed by water had extracted antioxidants more powerfully $(\mathrm{P}<0.05)$ than ethanol from lettuce. On the other hand, water followed by ethanol $(\mathrm{P}<0.05)$ had extracted antioxidants more $(\mathrm{P}<0.05)$ powerfully than methanol from watercress and parsley.

In terms of DPPH scavenging expressed as mg vitamin C/ $\mathrm{ml}$ solvent and regardless of the extraction solvent, grape leaves $>$ mint $>$ watercress $>$ Jew's mallow $>$ lettuce $>$ parsley had the highest antioxidant capacity values. Within the same context and in terms of the extraction solvent, ethanol followed by methanol $(\mathrm{P}<0.05)$ extracted antioxidants more powerfully $(\mathrm{P}<0.05)$ than water from watercress. On the other hand, water followed by methanol $(\mathrm{P}<0.05)$ had extracted antioxidants more powerfully
$(\mathrm{P}<0.05)$ than ethanol from grape leaves, mint, Jew's mallow, and garden rocket. Methanol followed by water $(\mathrm{P}<0.05)$ had extracted antioxidants more powerfully $(\mathrm{P}<0.05)$ than ethanol from celery, lettuce, and parsley.

With reference to correlations, antioxidant content (measured by Folin-Ciocultaeu method) of the studied vegetables correlated highly significantly with antioxidant capacity (measured by DPPH assay as percentage of scavenging ( $\left.\mathrm{r}=0.883, \mathrm{P}=0.004^{* *}\right)$, as $\mathrm{mg}$ vitamin $\mathrm{C} / \mathrm{ml}\left(\mathrm{r}=0.857, \mathrm{P}=0.007^{* *}\right)$, and as $\mathrm{M}$ trolox by CUPRAC assay $\left(\mathrm{r}=0.958, \mathrm{P}=0.000^{* *}\right)$ ). Additionally, there was a highly significant agreement expressed as a highly significant correlation between antioxidant capacity values measured by CUPRAC and DPPH assay (as DPPH scavenging percentage ( $r=0.860, \mathrm{P}=0.005)$ and as mg vitamin $\mathrm{C} / \mathrm{ml}(\mathrm{r}=0.845, \mathrm{P}=0.008)$ ).

Table 4: Comparison between values of antioxidant content and capacity found in this research and those found in other reports.

\begin{tabular}{|c|c|c|c|c|}
\hline Variable & Vegetable & Value Found in this Research & Value Found in Other Reports & Reference \\
\hline \multirow[t]{9}{*}{$\begin{array}{l}\text { Antioxidant Content: } \\
\text { Total Polyphenol (Folin- } \\
\text { Cioculteau Method) }\end{array}$} & Celery & $\begin{array}{c}0.39126 \mathrm{M} \text { catechin } / 100 \mathrm{~g} \text {. This value } \\
\text { corresponds to } 22.8309 \mathrm{~g} \text { catechin } \\
\text { equivalent } / 100 \mathrm{~g} \text {. }\end{array}$ & $20.55 \mathrm{~g}$ catechin equivalent $/ 100 \mathrm{~g}$ & {$[15]$} \\
\hline & Celery & $\begin{array}{l}0.39126 \mathrm{M} \text { catechin } / 100 \mathrm{~g} \text {. This value } \\
\text { corresponds to } 11.36 \mathrm{ppm} \text { in fresh celery. }\end{array}$ & $18.43 p p m$ catechin in fresh celery. & {$[36]$} \\
\hline & Garden Rocket & $\begin{array}{l}2.7845 \mathrm{M} \text { catechin } / 100 \mathrm{~g} \text { fresh garden } \\
\text { rocket; a value which corresponds to } \\
\text { 80.8219mg catechin/g fresh garden rocket. }\end{array}$ & $\begin{array}{c}1.2 \mathrm{mg} \text { total poly phenols/g fresh } \\
\text { weight. }\end{array}$ & {$[28]$} \\
\hline & Grape Leaves & $\begin{array}{l}42.2247 \mathrm{M} \text { catechin/100g fresh grape } \\
\text { leaves; a value which corresponds to } \\
1.226 \mathrm{mg} \text { catechin/g fresh grape leaves. }\end{array}$ & $\begin{array}{l}19.8 \text { and } 22.8 \mathrm{mg} \text { catechin/g fresh } \\
\text { grape leaves by extraction with } \\
80 \% \text { ethanol and } 80 \% \text { acetone } \\
\text { respectively. }\end{array}$ & {$[30]$} \\
\hline & Lettuce & $\begin{array}{c}1.1700 \mathrm{M} \text { catechin } / 100 \mathrm{~g} \text { fresh lettuce; } \\
\text { a value which corresponds to } 3.39 \mathrm{mg} \\
\text { catechin } / 100 \mathrm{~g} \text { fresh lettuce. }\end{array}$ & $\begin{array}{l}4.85 \mathrm{mg} \text { gallic acid } \\
\text { equivalent/100g fresh weight. }\end{array}$ & {$[19]$} \\
\hline & Mint & $\begin{array}{l}48.9688 \mathrm{M} \text { catechin } / 100 \mathrm{~g} \text { fresh mint; a } \\
\text { value which corresponds to } 101.52 \mathrm{mg} / \mathrm{g} \\
\text { fresh leaves. }\end{array}$ & $\begin{array}{l}25.62 \mathrm{mg} \text { catechin equivalent/g } \\
\text { fresh leaves. }\end{array}$ & {$[24]$} \\
\hline & Parsley & $\begin{array}{l}10.1102 \mathrm{M} \text { catechin } / 100 \mathrm{~g} \text { corresponds to } \\
2.098 \mathrm{~g} \text { catechin equivalent } / 100 \mathrm{~g} \text {. }\end{array}$ & 3.698g catechin equivalent/100g. & {$[15]$} \\
\hline & Jew's mallow & $\begin{array}{l}16.8973 \mathrm{M} \text { catechin/100g fresh sample. } \\
\text { Assuming } 82.4 \% \text { moisture content }[42,43] \\
\text { our value corresponds to } 27.867 \mathrm{mg} \\
\text { catechin/g dry Jews mallow. }\end{array}$ & 16.54 mg gallic acid/g dry weight. & {$[23]$} \\
\hline & Watercress & $\begin{array}{l}2.3643 \mathrm{M} \text { catechin } / 100 \mathrm{~g} \text { fresh watercress. } \\
\text { Assuming } 95.11 \% \text { moisture content [44]; } \\
\text { our value corresponds to } 140.6274 \mathrm{mg} \\
\text { catechin equivalent/g dry water cress. }\end{array}$ & $\begin{array}{l}217.14 \text { mg gallic acid equivalent/g } \\
\text { dry water cress. }\end{array}$ & {$[33]$} \\
\hline \multirow[t]{4}{*}{$\begin{array}{l}\text { Antioxidant Content: Total } \\
\text { Flavonoid Content }\end{array}$} & Celery & $\begin{array}{l}0.3378 \mathrm{M} \text { rutin } / 100 \mathrm{~g} \text { fresh celery. } \\
\text { This value corresponds to } 29.3601 \mathrm{mg} \\
\text { rutin } / 100 \mathrm{~g} \text { fresh celery }\end{array}$ & $\begin{array}{l}4.51 \mathrm{mg} \text { total flavonoids } / 100 \mathrm{~g} \\
\text { edible celery }\end{array}$ & {$[25]$} \\
\hline & Garden Rocket & $\begin{array}{l}0.4602 \mathrm{M} \text { rutin/100 g fresh watercress. } \\
\text { Assuming } 95.11 \% \text { moisture content [44]; } \\
\text { our value corresponds to } 575.7403 \mathrm{mg} \\
\text { rutin/g dry weight. }\end{array}$ & $0.82 \mu \mathrm{g}$ rutin/g dry weight. & [29] \\
\hline & Grape Leaves & $\begin{array}{c}0.8530 \mathrm{M} \text { rutin } / 100 \text { sample; a value which } \\
\text { corresponds to } 8.362 \mathrm{~g} \text { rutin/L extraction } \\
\text { solution. }\end{array}$ & $\begin{array}{l}56.38 \text { and } 100.08 \mathrm{mg} \text { rutin/L } \\
\text { grape leaves extracts of May and } \\
\text { September respectively. }\end{array}$ & {$[31]$} \\
\hline & Lettuce & $\begin{array}{c}0.0255 \mathrm{M} \text { rutin } / 100 \mathrm{~g} \text { fresh lettuce; a value } \\
\text { which corresponds to } 1.55 \mathrm{mg} \text { rutin/g fresh } \\
\text { sample. }\end{array}$ & $4.24 \mu \mathrm{g}$ rutin/g fresh lettuce. & {$[32]$} \\
\hline
\end{tabular}




\begin{tabular}{|c|c|c|c|c|}
\hline & Mint & $\begin{array}{l}2.438 \mathrm{M} \text { rutin } / 100 \mathrm{~g} \text {. This value corresponds } \\
\text { to } 148.844 \mathrm{mg} \text { rutin/g fresh sample. }\end{array}$ & $\begin{array}{l}\text { The amount of mint flavonoids is } \\
60.48 \mathrm{mg} / 100 \mathrm{~g} \text { fresh leaves. }\end{array}$ & [25] \\
\hline & Mint & $\begin{array}{l}2.438 \mathrm{M} \text { rutin } / 100 \mathrm{~g} \text {. This value corresponds } \\
\text { to } 0.02438 \mathrm{mg} \text { rutin/g fresh sample. }\end{array}$ & $0.01 \mathrm{mg}$ rutin/g fresh leaves. & [39] \\
\hline & Parsley & $\begin{array}{l}3.7390 \mathrm{M} \text { rutin } / 100 \mathrm{~g} \text {; a value which } \\
\text { corresponds to } 23.1937 \mathrm{mg} \text { rutin } / 100 \mathrm{~g} \text {. }\end{array}$ & $4.32 \mathrm{mg}$ rutin $/ 100 \mathrm{~g}$ fresh parsley. & {$[40]$} \\
\hline & Jew's mallow & $\begin{array}{l}0.4435 \mathrm{M} \text { rutin/100g fresh Jews mallow; } \\
\text { a value which corresponds to } 0.154 \mathrm{mg} \\
\text { rutin/g dry sample. }\end{array}$ & $\begin{array}{l}0.33 \mathrm{mg} \text { rutin/g dry Jew's mallow } \\
\text { stems. }\end{array}$ & [38] \\
\hline & Watercress & $\begin{array}{c}0.1990 \mathrm{M} \text { rutin/100g fresh watercress. } \\
\text { Assuming } 95.11 \% \text { moisture content [44]; } \\
\text { our value corresponds to } 248.962 \mu \mathrm{g} \text { rutin/g } \\
\text { dry weight. }\end{array}$ & $126.57 \mu \mathrm{g}$ rutin/g dry weight. & [29] \\
\hline \multirow[t]{9}{*}{$\begin{array}{l}\text { Antioxidant Capacity: } \\
\text { CUPRAC Assay }\end{array}$} & Celery & $\begin{array}{l}1.3059 \mathrm{M} \text { trolox/100g; a value which } \\
\text { corresponds to } 1.747 \mu \mathrm{mol} \mathrm{TE} / \mathrm{ml} \text {. }\end{array}$ & $\begin{array}{l}9 \text { and } 11 \mu \mathrm{mol} \mathrm{TE} / \mathrm{ml} \text { and } 0.25 \\
\text { and } 3.5 \mu \mathrm{mol} \mathrm{TE} / \mathrm{ml} \text { broth of fresh } \\
\text { leaves and stalk respectively. }\end{array}$ & {$[21]$} \\
\hline & Garden Rocket & $\begin{array}{l}3.2696 \mathrm{M} \text { trolox/100 g fresh garden rocket; } \\
\text { a value which corresponds to } 18.16 \mu \mathrm{mol} \\
\text { TE/g fresh garden rocket. }\end{array}$ & $\begin{array}{l}8.18 \text { and } 32.08 \mu \mathrm{mol} \mathrm{TE} / \mathrm{g} \text { fresh } \\
\text { garden rocket by DPPH and ORAC1 } \\
\text { assays for antioxidant capacity. }\end{array}$ & {$[29]$} \\
\hline & Grape Leaves & $\begin{array}{l}30.0380 \mathrm{M} \text { trolox } / 100 \mathrm{~g} \text { fresh sample; a } \\
\text { value which corresponds to } 3.0038 \mathrm{mmol} \\
\text { trolox/g fresh sample. }\end{array}$ & $0.12 \mathrm{mmol}$ trolox $/ \mathrm{g}$ fresh sample. & {$[30]$} \\
\hline & Lettuce & $\begin{array}{c}\text { our value for lettuce corresponds to } \\
675.507 \mu \mathrm{mol} T \mathrm{TE} / 100 \mathrm{~g} .\end{array}$ & $\begin{array}{l}491 \mu \mathrm{mol} \text { TE } / 100 \mathrm{~g} \text { fresh lettuce } \\
\text { (ORAC method). }\end{array}$ & [17] \\
\hline & Mint & $\begin{array}{c}22.8008 \mathrm{M} \text { trolox } / 100 \mathrm{~g} \text { fresh leaves. } \\
\text { Assuming } 69.6 \% \text { moisture content [44], our } \\
\text { value corresponds to } 7500.2632 \mu \mathrm{mol} / 100 \\
\text { g dry weight. }\end{array}$ & $386 \mu \mathrm{mol}$ trolox/100g dry weight. & {$[27]$} \\
\hline & Parsley & $\begin{array}{l}\text { Our value corresponds to } 18.508 \mathrm{~g} \\
\text { trolox/100g fresh parsley. }\end{array}$ & $\begin{array}{l}987.51 \mathrm{mg} \text { trolox } / 100 \mathrm{~g} \text { fresh } \\
\text { parsley. }\end{array}$ & [39] \\
\hline & Parsley & $\begin{array}{c}\text { Assuming } 25.3 \% \text { dry matter. Our value } \\
\text { corresponds to } 1623.715 \mu \mathrm{mol} \text { TE/100g dry } \\
\text { matter. }\end{array}$ & $\begin{array}{l}340.68 \mu \mathrm{mol} \mathrm{TE} / 100 \mathrm{~g} \text { dry matter } \\
\text { (average of } 2 \text { methods; DPPH and } \\
\text { ABTS2). }\end{array}$ & [26] \\
\hline & Jew's mallow & $\begin{array}{l}13.3043 \mathrm{M} \text { trolox/100 g fresh sample; a } \\
\text { value which corresponds to } 75.593 \mu \mathrm{mol} \\
\text { trolox/g dry weight. }\end{array}$ & $\begin{array}{l}\text { antioxidant capacity by ABTS } \\
\text { method to be } 139.55 \mu \text { mol trolox/g } \\
\text { dry weight. }\end{array}$ & [23] \\
\hline & Watercress & $\begin{array}{l}2.658 \mathrm{M} \text { trolox } / 100 \mathrm{~g} \text { fresh garden rocket; } \\
\text { a value which corresponds to } 14.767 \mu \mathrm{mol} \\
\mathrm{TE} / \mathrm{g} \text { fresh garden rocket. }\end{array}$ & $\begin{array}{l}7.76 \text { and } 32.92 \mu \mathrm{mol} \mathrm{TE} / \mathrm{g} \text { fresh } \\
\text { watercress by DPPH and ORAC } \\
\text { assays for antioxidant capacity. }\end{array}$ & {$[29]$} \\
\hline \multirow[t]{6}{*}{$\begin{array}{l}\text { Antioxidant Capacity: DPPH } \\
\text { Scavenging Percentage }\end{array}$} & Celery & Our values ranged between $\sim 5$ and $\sim 17 \%$. & $\begin{array}{l}\text { DPPH\% scavenging capacity value } \\
\text { of the essential oils of celery herb } \\
\text { and seeds to be } 56.68 \text { and } 74.35 \\
\text { respectively. }\end{array}$ & [34] \\
\hline & Garden Rocket & $\begin{array}{l}\text { Our values ranged between } \sim 11 \text { and } \\
\sim 17.8 \% .\end{array}$ & $\begin{array}{l}20-60 \% \text { inhibition of alcohol and } \\
\text { hydro alcohol extracts of garden } \\
\text { rocket (range is due to the extract } \\
\text { concentration; ranged between } \\
\quad 10-320 \mu \mathrm{g} / \mathrm{ml} \text { ). }\end{array}$ & {$[20]$} \\
\hline & Grape Leaves & $\begin{array}{l}\text { Ranged between } \sim 35 \% \text { and } \sim 88 \% \text {. } \\
\text { Referring to our raw data records, our } \\
\text { samples were purchased in August. }\end{array}$ & $\begin{array}{l}61.69 \% \text { and } 70.32 \% \text { for May and } \\
\text { September vine leaves. }\end{array}$ & {$[31]$} \\
\hline & Lettuce & Our values ranged between $\sim 9$ and $\sim 28 \%$. & 80.9 in 12 US lettuce varieties. & [35] \\
\hline & Mint & Our values ranged from $\sim 25 \%$ to $\sim 88 \%$. & $\begin{array}{l}18 \text { and } 35 \text { for mint stem and } \\
\text { leaves respectively (diethylether } \\
\text { extracts). }\end{array}$ & {$[16]$} \\
\hline & Parsley & Our values ranged between $\sim 6$ and $\sim 14 \%$. & $\begin{array}{l}20-30 \% \text { of DPPH radical } \\
\text { scavenging capacity in } \\
\text { field and aeroponic grown } \\
500 \mu \mathrm{g} / \mathrm{ml} \text { parsley extracted } \\
\text { dimethylsulfoxide extracts. }\end{array}$ & [23] \\
\hline
\end{tabular}




\begin{tabular}{|l|l|c|c|c|}
\hline & Jew's mallow & $\begin{array}{c}21.5090 \% \text {. Assuming 82.4\% moisture } \\
\text { content [42], this value corresponds to } \\
129.5723 \% .\end{array}$ & $52.29 \%$ in the dry weight. \\
\hline & Watercress & Our values ranged between $\sim 23$ and $\sim 39 \%$ & $\begin{array}{c}60-80 \% \text { DPPH radical scavenging } \\
\text { capacity of the aqueous and } \\
\text { methanolic extracts of watercress } \\
\text { stem and leaves. }\end{array}$ \\
[37]
\end{tabular}

The antioxidant content and capacity values of the studied vegetables have been published previously. Table 4 shows a comparison between values of antioxidant content and capacity found in this research and those found in other reports. The previously published values were sometimes comparable with the values found in this research [15-23], sometimes much higher [24-29] or much lower [23,30-39]. The differences found are probably attributed to the differences in the extraction solvent type, treatment, and concentration extraction methods, experimental methodology, plant growing conditions as Jordan has variation in agricultural environment because it meets the Mediterranean, Irano-turanian, and Saharo-Arabian region [40], parts of the analyzed plant, and the experimental standardization conditions. The expression of antioxidant capacity (measured by DPP radical scavenging assay) as mg of vitamin C is another method for expression that will add a value to the scientific antioxidant capacity database.

Many scientists agree that there is no single best method for measuring antioxidant content and capacity [2]. Nonetheless, in either method for determining antioxidant content; grape leaves, mint, and parsley showed the highest 3 levels of antioxidant contents. Furthermore, grape leaves, mint, and watercress showed the highest levels of antioxidant capacities. With reference to correlations; similar to the results found by Apak et.al. [2], the highly significant correlation between the antioxidant content (measured by Folin-Ciocultaeu method) of the studied vegetables with antioxidant capacity (measured by DPPH as well as CUPRAC) assays is not surprising since the antioxidants measured by Folin-Ciocaltaeu method contributed well to the antioxidant capacity. Similar to the results of Kaur \& Mondal [41], there was no correlation between the antioxidant content measured by total flavonoid content and antioxidant capacity owing that the antioxidant capacity is not solely due to the total flavonoids content. The highly significant agreement expressed as a highly significant correlation between antioxidant capacity values measured by CUPRAC and DPPH assays is not surprising as both assays measure the same reaction kinetics (ET-assays).

\section{Conclusion}

Significant differences $(\mathrm{P}<0.05)$ were found among vegetables extracts in terms of antioxidant contents and capacities. Also, we find highly significant $\left(\mathrm{P}<0.001^{* *}\right)$ correlations between antioxidant content and capacity values. This study is limited by the types of extracts used and the extraction methods as well as the treatment of the vegetables (as the vegetables were fresh). However, this study is probably the first study which analyzed and compared three extracts types of the eight fresh green leafy vegetables and will start a database for the antioxidant content and capacity of Jordanian vegetables.

\section{Acknowledgement}

The authors would like to thank the Deanship of Academic Research at The University of Petra for financially supporting this research [Grant number 5/4/2015].

\section{Conflict of Interest}

The authors declare that there is no conflict of interest among them.

\section{References}

1. Halliwell B, Gutteridge JC (1995) The definition and measurement of antioxidants in biological systems. Free Radic Biol Med 18(1): 125-126.

2. Apak R, Güçlü K, Demirata B, Özyürek M, Çelik S, Bektaşoğlu B, et al. (2007) Comparative evaluation of various total antioxidant capacity assays applied to phenolic compounds with the CUPRAC assay. Molecules 12(7): 1496-1547.

3. Tawaha K, Alali F, Gharaibeh M, Mohammad M, El-Elmat T (2007) Antioxidant activity and total phenolic content of selected Jordanian plant species. Food Chemistry 104(4): 1372-1378.

4. Al-Mustafa A, Al-Thunaibat O (2008) Antioxidant activity of some Jordanian medicinal plants used traditionally for treatment of diabetes. Pak J Biol Sci 11(3): 351-368.

5. Al-Dabbas M, Saleh M, Al-Ismail K (2015) Preservation methods impacted phenolic flavonoid and carotenoid contents and antioxidant activities of carrots (Daucus carota L.). Journal of Food Processing and Preservation 39(6): 1618-1625.

6. Al-Eisawi D, Takruri H (1989) A checklist of wild edible plants in Jordan. Arab Gulf Journal of Scientific Research, Agricultural and Biological Sciences B7(1): 79-102.

7. Tukan S, Takruri H, Al-Eisawi D (1998) The use of wild edible plants in the Jordanian diet. Int J Food Sci Nutr 49(3): 225-235.

8. Al-Kurd R, Takruri H, Al-Sayyed H (2008) Tannin content of selected plants used in Jordan. Jordan Journal of Agricultural Sciences 4(3): 265-273.

9. Al-Sayyed H, Takruri H, Shomaf M (2014) The effect of date palm fruit (Phoenix dactylifera L.) on 7, 12-dimethylbenz ( $\alpha$ ) anthracene (DMBA)induced mammary cancer in rats. Research Opinions in Animal and Veterinary Sciences 4(1): 11-18.

10. Luterotti S, Bicanic D, Pozgaj R (2006) New simple spectrophotometric assay of total carotenes in margarines. Anal Chim Acta 573-574: 466473.

11. Agbor G, Vinson J, Donnelly P (2014) Folin-Ciocalteau reagent for polyphenolic assay Int J Food Sci Nutr Diet 3(8): 147-156.

12. Pękal A, Pyrzynska K (2014) Evaluation of aluminium complexation reaction for flavonoid content assay. Food Analytical Methods 7(9): 1776-1782. 
13. Molyneux P (2003) The use of the stable radical diphenylpicrylhydrazyl (DPPH) for estimating antioxidant activity. Songklanakarin J Sci Technol 26(2): 211-219.

14. Laerd Statistics (2018) Statistical tutorials and software guides.

15. Alezandro M, Youn M, Lajolo F, Genovese M (2011) Commercial spices and industrial ingredients: evaluation of antioxidant capacity and flavonoids content for functional foods development. Ciência e Tecnologia de Alimentos 31(2): 527-533.

16. Al-Juhaimi F, Ghafoor K (2011) Total phenols and antioxidant activities of leaf and stem extracts from coriander, mint and parsley grown in Saudi Arabia. Pak J Bot 43(4): 2235-2237.

17. Mikami-Konishide I, Murakami S, Nakanishi K, Takahashi Y, Yamaguchi M, et al. (2013) Antioxidant Capacity and Polyphenol Content of Extracts from Crops Cultivated in Japan, and the Effect of Cultivation Environment. Food Science and Technological Research 19(1): 69-79.

18. Cirlini M, Mena P, Tassotti M, Herrlinger K, Nieman K, Dall'Astam C, et al. (2016) Phenolic and Volatile Composition of a Dry Spearmint (Mentha spicata L.) Extract. Molecules 21(8): E1007-E1022.

19. Gan Y, Arzina A (2016) Antioxidant properties of selected varieties of lettuce (Lactuca sativa L.) commercially available in Malaysia. International Food Research Journal 23(6): 2357-2362.

20. Kishore L, Kaur N, Singh R (2016) Evaluation of antioxidant activity and total phenolic content of Eruca sativa L. seeds. International Journal of Toxicological and Pharmacological Research 8(3): 146-151.

21. Filiz E, Korkmaz N, Budak N, Seydim A, Guzel Z (2017) Antioxidant activity and phenolic acid content of selected vegetable broths. Czech J Food Sci 35(6): 469-475.

22. Chandra S, Khan S, Avula B, Lata H, Yang M, et al. (2014) Assessment of total phenolic and flavonoid content, antioxidant properties, and yield of aeroponically and conventionally grown leafy vegetables and fruit crops: a comparative study. Evid Based Complement Alternat Med. P. $1-9$.

23. Youssef M, Mokhtar S, Morsy N (2014) Effect of hot air drying variables on phytochemicals and antioxidant capacity of Jew's Mallow (Corchorus olitorius L.) leaves. Journal of Food Sciences, Suez Canal University 2(1): $1-8$.

24. Kanatt S, Chander R, Sharma A (2007) Antioxidant potential of mint (Mentha spicata L.) in radiation-processed lamb meat. Food Chemistry 100(2): 451-458.

25. United States Department of Agriculture (USDA) (2011) USDA Database for the Flavonoid Content of Selected Foods (Release 3). USA: USDA. pp. 159.

26. Priecina L, Karklina D (2014) Natural antioxidant changes in fresh and dried spices and vegetables. International Journal of Nutrition and Food Engineering 8(5): 492-496.

27. Luminița P (2015) Comparative evaluation of antioxidant capacity of herbal plants by different methods. Journal of Horticulture, Forestry and Biotechnology 19(4): 9-12.

28. Spadafora N, Amaro A, Pereira M, Müller C, Pintado M, et al. (2016) Multi-trait analysis of post-harvest storage in rocket salad (Diplotaxis tenuifolia) links sensorial, volatile and nutritional data. Food Chem 211: 114-123.
29. Li Z, Lee H, Liang X, Liang D, Wang Q, et al. (2018) Profiling of phenolic compounds and antioxidant activity of 12 cruciferous vegetables. Molecules 23(5): E1139.

30. Amarowicz R, Narolewska O, Karamać M, Kosińska A, Weidner S (2008) Grapevine leaves as a source of natural antioxidants. Pol J Food Nutr Sci 58(1): 73-78.

31. Katalinić V, Generalić I, Skroza D, Ljubenkov I, Teskera A, et al. (2009) Insight in the phenolic composition and antioxidative properties of Vitis vinifera leaves extracts. Croat J Food Sci Technol 1(2): 7-15.

32. Khanam U, Oba S, Yanase E, Murakami Y (2012) Phenolic acids, flavonoids and total antioxidant capacity of selected leafy vegetables. Journal of Functional Foods 4(4): 979-987.

33. Boligon A, Janovik V, Boligon A, Pivetta C, Pereira R, et al. (2013) HPLC analysis of polyphenolic compounds and antioxidant activity in Nasturtium officinale. International Journal of Food Properties 16(1): 61-69.

34. Hassanen N, Eissa A, Hafez S, Mosa E (2015) Antioxidant and antimicrobial activity of celery (Apium graveolens) and coriander (Coriandrum sativum) herb and seed essential oils. Int J Curr Microbiol App Sci 4(3): 284-296.

35. Liua X, Ardob S, Bunninga M, Parryb J, Zhoub K, et al. (2007) Total phenolic content and DPPH radical scavenging activity of lettuce (Lactuca sativa L.) grown in Colorado. LWT-Food Science and Technology 40(3): 552-557.

36. Sorour M, Hassanen N, Ahmed M (2015) Natural antioxidant changes in fresh and dried celery (Apium graveolens). American Journal of Energy Engineering 3: 12-16.

37. Zeb A (2015) Phenolic profile and antioxidant potential of wild watercress (Nasturtium officinale L.). Springerplus 4: 714-721.

38. Arfa N, El-Desouky A, Bahlol H, Soliman A (2017) Utilization of Jew's mallow stems as a natural source of dietary fiber in pan bread product. Annals of Agric Sci, Moshtohor 55(2): 333-342.

39. Nour V, Trandafir I, Cosmulescu S (2017) Bioactive compounds, antioxidant activity and nutritional quality of different culinary aromatic herbs. Not Bot Horti Agrobo 45(1): 179-184.

40. Qura'n S (2010) Ethnobotanical and ecological studies of wild edible plants in Jordan. Libyan Agriculture Research Center Journal International 1: 231-243.

41. Kaur S, Mondal P (2014) Study of total phenolic and flavonoid content, antioxidant activity and antimicrobial properties of medicinal plants. Journal of Microbiology and Experimentation 1(1): 1-6.

42. Pelltte P, Shadervian S (2013) Food composition tables for the use in the Middle East. ( $2^{\text {nd }}$ edn), Beirut, Lebanon: American University of Beirut Press, USA, pp. 126.

43. U.S. Department of Agriculture, Agricultural Research Service (2018) USDA Nutrient Database for Standard Reference, Release 18.

44. Kumar A, Kumar V, Khan K, Kumar A (2017) Experimental investigation on drying of mint leaves (M. Pulegium) in solar tunnel dryer. Int J Pure App Biosci 5(2): 682-689. 
This work is licensed under Creative Commons Attribution 4.0 License

DOI: 10.19080/ARTOAJ.2019.19.556102

\section{Your next submission with Juniper Publishers} will reach you the below assets

- Quality Editorial service

- Swift Peer Review

- Reprints availability

- E-prints Service

- Manuscript Podcast for convenient understanding

- Global attainment for your research

- Manuscript accessibility in different formats

( Pdf, E-pub, Full Text, Audio)

- Unceasing customer service

Track the below URL for one-step submission https://juniperpublishers.com/online-submission.php 